\title{
Infections bactériennes invasives chez l'enfant drépanocytaire à Bamako, Mali.
}

\section{Invasive bacterial infections occurring to sickle cell children in Bamako, Mali}

Diakité $\mathrm{AA}^{1,2}$, Balilé $\mathrm{H}^{1}$, Konaté $\mathrm{I}^{2}$, Traoré $\mathrm{A}^{1}$, Traore $\mathrm{NL}^{1}$, Dicko $\mathrm{FT}^{1}$, Touré $\mathrm{A}^{1}$, Diakité $\mathrm{F}$ $\mathrm{L}^{1}$, Togo $\mathrm{B}^{1,2}$, Sylla $\mathrm{M}^{1,2}$, Diallo $\mathrm{S}^{2,3}$.

${ }^{1}$ Département de pédiatrie, CHU Gabriel Touré, Bamako, Mali

${ }^{2}$ Faculté de médecine et d'odontostomatologie

${ }^{3}$ Centre d'infectiologie Charles MERIEUX

\section{Auteur correspondant:}

Dr Abdoul Aziz Diakité

Email : docabdela@yahoo.fr Tel : + 22366744956

\section{Résumé :}

Les infections sont responsables d'une part importante de la morbidité et de la mortalité chez l'enfant drépanocytaire. Notre objectif était d'étudier le profil clinique et bactériologique des infections bactériennes de l'enfant drépanocytaire dans le service de pédiatrie du CHU Gabriel Touré. Matériel et méthodes: Nous avons réalisé une étude rétrospective sur 25 dossiers d'hospitalisation d'enfants drépanocytaires fébriles ayant bénéficié d'une hémoculture sur une période de 5 ans (2005-2010). Nous avons analysé les caractéristiques cliniques et bactériologiques des enfants ayant une hémoculture positive. Résultats: Dix des 25 hémocultures réalisées étaient positives. La tranche d'âge de 0-5 ans était la plus touchée (60\%). Le suivi était irrégulier pour 7 enfants et 6/10 n'avaient pas reçu le vaccin anti pneumococcique. Sur le plan clinique, 5 cas de sepsis, une infection pulmonaire et 4 cas infections ostéo articulaires ont été diagnostiquées. La goutte épaisse était positive pour 4 patients. Les germes retrouvés ont été : Salmonella enterica sérotype Typhi (4 fois), Streptococcus pneumoniae (4 fois), Staphylococcus aureus (1fois), Enterobacter cloacae (1fois) ont été isolés. Salmonella enterica sérotype Typhi a été isolé dans 3 cas d'infections ostéo-articulaires et un cas de sepsis, Streptococcus pneumoniae était en cause dans l'infection pulmonaire et dans un cas de sepsis. L'antibiothérapie probabiliste dans la majorité des cas était basée sur les céphalosporines de troisième génération. L'évolution a été 
favorable dans $80 \%$ des cas. Conclusion : Les complications infectieuses de la drépanocytose peuvent être graves. La disponibilité de l'hémoculture doit nous permettre de caractériser le profil bactériologique dans notre contexte.

Mots clés : drépanocytose, enfant, infections.

\section{Summary:}

Infections are responsible for a large share of morbidity and mortality in sickle cell children. Our objective was to study the clinical and bacteriological profile of bacterial infections of sickle cell child in the pediatric ward of the University Hospital Gabriel Touré. Methods: We performed a retrospective study of 25 hospital records of febrile children with sickle cell disease who received a blood culture over a period of 5 years (2005-2010). We analyzed the clinical and bacteriological characteristics of children with positive blood culture. Results: Ten of the 25 blood cultures were positive. The age group of 0-5 years were the most affected (60\%). The monitoring was irregular for children 7 and $6 / 10$ had not received the anti pneumococcal vaccine. Clinically, 5 cases of sepsis, pulmonary infection and 4 cases articular bone infections were diagnosed. Thick smear was positive for 4 patients. The germs found were: Salmonella enterica sérotype Typhi (4 times), Streptococcus pneumoniae (4 times), Staphylococcus aureus (1 time), Enterobacter cloacae (1 time) were isolated. Salmonella enterica serotype Typhi was isolated in 3 cases of bone and joint infections and sepsis, Streptococcus pneumoniae was involved in a lung infection and sepsis. The empirical antibiotic therapy in the majority of cases was based on the third-generation cephalosporins. The evolution was favorable in $80 \%$ of cases. Conclusion: Infectious complications of sickle cell disease can be severe. The availability of blood culture should allow us to characterize the bacterial profile in our context.

Keywords: child, sickle cell anemia, infections. 


\section{Introduction}

La drépanocytose est classée au $4^{\text {ème }}$ rang des maladies prioritaires de l'OMS après le paludisme, le VIH et la tuberculose. Elle s'observe dans le monde entier avec une prévalence accrue en Afrique [1]. Elle se traduit cliniquement par une anémie hémolytique chronique. Son évolution est émaillée d'épisodes de déglobulisation aiguë et d'une asplénie fonctionnelle responsable d'une susceptibilité aux infections à germes encapsulés [2]. Les infections sont responsables d'une part importante de la morbidité et la mortalité de la drépanocytose chez l'enfant. La plus forte incidence des infections est observée dans les premières années de vie [3]. Sur le continent africain $5 \%$ des enfants de moins de cinq ans décèdent de drépanocytose. Cette proportion passe à plus de $9 \%$ en Afrique de l'Ouest. [4]. Des statistiques récentes du Nigeria montrent que $50 \%$ des enfants atteints de drépanocytose décèdent avant l'âge de 5 ans [5]. La fréquence des accidents infectieux diminue avec l'âge, mais le risque persiste toute la vie. Les méningites et les septicémies sont les infections les plus graves chez les enfants drépanocytaires. Ces infections peuvent mettre en jeu le pronostic vital ou causer des séquelles [3]. Les ostéomyélites sont des complications infectieuses les plus courantes. Tous les os peuvent être atteints et les localisations plurifocales ne sont pas rares [6]. L'absence d'étude antérieure sur le profil infectieux chez l'enfant drépanocytaire dans le service nous a amené à étudier cet aspect dans notre cohorte.

\section{Patients et méthode:}

Nous avons réalisé une étude rétrospective sur 25 dossiers d'hospitalisation d'enfants drépanocytaires fébriles ayant bénéficié d'une hémoculture sur une période de 5 ans (2005-2010). Nous avons analysé les caractéristiques cliniques et bactériologiques des enfants drépanocytaires ayant une hémoculture positive. Dans cette étude tous les enfants 
inclus avaient une forme majeure de drépanocytose.

Pour cette étude, ont été considérés comme :

Drépanocytaires SS $=$ taux d'hémoglobine $\mathrm{S} \geq 75 \%$

Drépanocytaires $\mathrm{S} \beta^{0}$ thalassémie $=\operatorname{taux}$ d'hémoglobine A2 > 3,5\% avec absence de l'hémoglobine A1; présence d'hémoglobine $\mathrm{S}$

Drépanocytaires $S \beta^{+}$thalassémie $=$taux d'hémoglobine A2 $>3,5 \%$ avec présence de l'hémoglobine A1; présence d'hémoglobine S.

Suivi régulier : tout malade venant à toutes les visites systématiques ;

Suivi irrégulier : tout malade ayant manqué au moins une visite ;

Septicémies $=$ signes de sepsis avec isolement de germe pathogène à l'hémoculture.

Pneumopathie $=$ râles crépitant avec ou sans détresse respiratoire et /ou opacités à la radiographie pulmonaire.
La cholécystite $=$ signe de Murphy et /ou l'échographie abdominale objectivant une cholécystite

Atteintes ostéo-articulaires (ostéomyélite, arthrite $)=$ tuméfaction douloureuse des membres avec impotence fonctionnelle et fièvre et/ou signes radiologiques d'atteinte ostéo-articulaires.

Hyperleucocytose = leucocytes > $10.000 / \mathrm{mm}^{3}$

$\mathrm{CRP}$ positive $=\geq 7 \mathrm{mg} / 1$

Vaccins hors PEV = R.O.R (anti-rougeole, anti- oreillon, anti-rubéole), Pneumo 23 (anti- pneumococcique, Typhium Vi (contre la fièvre typhoïde), méningo $\mathrm{A}+\mathrm{C}$ (anti-meningococcique $\mathrm{A}+\mathrm{C}$ ), Vaxigrip (vaccin contre grippe), Avaxim 80 (vaccin contre l'hépatite A)

\section{Caractéristiques sociodémographiques} et cliniques

Age ; Sexe ; la provenance ; la profession des parents.

Le suivi ; la scolarité ; le statut vaccinal ; paramètres anthropométriques; les signes cliniques ; les pathologies associées. 


\section{Caractéristiques para-cliniques}

Electrophorèse de l'hémoglobine; la numération formule sanguine ; la proteine C Reactive (CRP); la goutte épaisse; l'hémoculture.

\section{Aspects thérapeutiques et évolutifs}

Les molécules utilisées; devenir des malades.

L'analyse et le traitement des données ont été réalisés sur la version 12.0 du logiciel SPSS.

\section{RESULTATS}

Pendant la période d'étude sur 25 dossiers d'hospitalisation d'enfants drépanocytaires fébriles ayant bénéficié d'une hémoculture sur une période de 5 ans (2005-2010) nous avons retrouvé 10/25 hémocultures positives. Au cours de cette période il y avait 500 enfants drépanocytaires suivis ce qui fait une prévalence des infections invasives de $5 \%$.

\section{Caractéristiques des enfants :}

La tranche d'âge de 0-5 ans était la plus touchée $(60 \%)$. L'âge moyen des enfants était de 2ans avec des extrêmes 4 mois et
14 ans. Le sexe masculin était le plus représenté avec une fréquence de $52 \%$, le sexe ratio est de 1, 06. Plus de la moitié des malades présentaient une dénutrition légère $(52 \%)$.

Le suivi était irrégulier pour 7 enfants et 6/10 n'avaient pas reçu le vaccin anti pneumococcique.

Sur le plan clinique, 5 cas de sepsis, une infection pulmonaire et 4 cas infection ostéo articulaire ont été diagnostiqués. Les septicémies et les pneumopathies ont été plus retrouvées chez les sujets SS et S$\beta$ thalassémiques. Les complications ostéoarticulaires (ostéomyélite et arthrite) ont été retrouvées dans toutes les formes. Le tableau I regroupe les diagnostics et les formes drépanocytoses chez les enfants.

Sur le plan para clinique les drépanocytaires SS avaient un taux d'hémoglobine compris entre 4-6g/dl avec une moyenne $4 \pm 1,5 \mathrm{~g} / \mathrm{dl}$ suivis des S- $\beta$ th avec un taux $7-9 \mathrm{~g} / \mathrm{dl}$ et moyenne 6 $\pm 1,8 \mathrm{~g} / \mathrm{dl}$. Nous avons mise en évidence de 
trophozoites de Plasmodium chez 4

patients.

\section{Discussion}

Les germes retrouvés ont été : Salmonella

Au cours de cette étude rétrospective dans enterica sérotype Typhi (4 fois), le département de pédiatrie qui a concerné Streptococcus pneumoniae (4 fois), Staphylococcus aureus (1fois), Enterobacter cloacae (1fois) ont été isolés. Salmonella enterica a été isolé dans 3 cas d'infections ostéo-articulaires et un cas de sepsis, Streptococcus pneumoniae était en cause dans l'infection pulmonaire et dans un cas de sepsis. L'hémoculture est revenue stérile dans $52 \%$ des cas. Le tableau II regroupe les germes retrouvés à l'hémoculture et les formes de drépanocytoses chez les enfants.

Aspects thérapeutiques et évolutifs des enfants

L'antibiothérapie probabiliste dans la majorité des cas était basée sur les céphalosporines de troisième génération. L'évolution été favorable dans $80 \%$ des cas. Le tableau III regroupe l'évolution des enfants en fonction des pathologies associées à la drépanocytose. 25 dossiers d'enfants sur une cohorte de 500 enfants suivis à l'unité de prise en charge drépanocytaire durant 5 ans. La prévalence des complications infectieuses était de $5 \%$ et 10 des 25 hémocultures réalisées étaient positives.

\section{-Caractéristiques des enfants :}

Nous avons constaté que les drépanocytaires mal ou pas suivis présentaient plus de complications infectieuses. Cette catégorie représentait les $2 / 3$ des malades de notre série. La tranche d'âge de 0-5 ans était la plus touchée $(60 \%)$. L'âge moyen des enfants était de 2 ans ,ce constat a été fait par plusieurs auteurs $[7,8,9]$. Selon Markus Schmugge [10], les crises vaso-occlusives récidivantes rendent la rate rapidement non fonctionnelle; soit qu'un choc circulatoire aigu entraîne une séquestration splénique soit que des vaso-occlusions moins 
importantes mais répétées provoquent une atrophie splénique. Une asplénie fonctionnelle était présente chez $30 \%$ des enfants de douze mois et chez $90 \%$ des enfants de six ans. C'est pour cette raison que le risque d'infection par des bactéries encapsulées est élevé à cet âge.

Tous les enfants hospitalisés ont été correctement vaccinés selon le programme élargi de vaccination (PEV), mais $60 \%$ d' entre eux n'ont pas reçu les vaccins hors PEV. Cela s'explique par le fait que l'administration de ces vaccins est corrélée au suivi médical et aux conditions socio-économiques des parents. $\mathrm{Au}$ Burkina Faso [11] la couverture vaccinale des enfants drépanocytaires variait de 5,7 $\%$ pour le vaccin anti-Hib à $65,8 \%$ pour le Pneumo 23. Les causes majeures de nonvaccination étaient l'ignorance et le coût prohibitif de ces vaccins quand l'information était connue. Les septicémies et les pneumopathies ont dominé notre série. A Bangui [12] le paludisme vient en tête avec 48 cas (39 \%) suivi des infections broncho-pulmonaires $(22,8 \%)$. Contrairement à notre série Sangaré $\mathrm{A}$ et al. [13] trouvent une prédominance des infections respiratoires soit $61 \%$ suivis d'accès palustre $48 \%$. Au Congo Brazzaville [14] la localisation bronchopulmonaire a été la plus observée dans $16,2 \%$ des cas, l'infection urinaire arrivait en seconde position avec 9,2\% des cas, l'infection des voies biliaires dans 7,8\% des cas et enfin l'ostéomyélite aiguë (OMA) n'était observée que dans 7\% des cas.

En Europe [15], le sepsis vient en tête (les germes incriminés sont généralement les salmonelles et Escherichia Coli pour les Gram négatif et le staphylocoque doré pour Gram positif)

Dans notre série l'accès palustre a été le plus observé chez les sujets SS. Les septicémies et les pneumopathies ont été plus observées chez les sujets SS suivis des S- $\beta$ Thal contrairement à Sangaré et al. [13] qui ont remarqué que les formes anémiques (SS et SF) présentent plus 
d'infections bactériennes, alors que les formes non anémiques (SC et S- $\beta$ thal) présentent plus d'accès palustre.

Les complications ostéo-articulaires ont été retrouvées dans toutes les formes.

Souna [16] dans sa série au Niger a trouvé que les formes homozygotes (SS) représentaient $59,1 \%$ des cas. Ce sont les crises vaso-occlusives fréquentes chez ces enfants qui entraînent l'anoxie puis l'ischémie et l'infarctus osseux, favorisant la greffe bactérienne, surtout au niveau de l'os spongieux, donc des métaphyses.

La seconde enfance et l'adolescence sont émaillées de crises douloureuses. Pendant cette période, débute la pathologie dégénérative tissulaire. Ces complications obligent à des consultations hospitalières et parfois à des hospitalisations, d'où des perturbations de la scolarité.

Au cours de l'étude les sujets SS ont été les plus nombreux avec une fréquence de $84 \%$ suivis des $\mathrm{S}-\beta$ thal $12 \%$, et SC $4 \%$. Ces résultats sont similaires à ceux retrouvés en Côte d'Ivoire par Sangaré et al [13]. Au TOGO H. TEKOU et al [17] dans une étude sur le profil des ostéomyélites hématogènes de l'enfant rapporte une prédominance des drépanocytaires SS $50,8 \%$ suivis des SC $32,3 \%$ et des AS 12,3\%. Les complications infectieuses sont ainsi plus fréquentes chez les sujets SS. L'hémolyse étant plus marquée chez ses sujets, cela favorise la séquestration splénique et augmente le risque d'infection contrairement aux formes moins anémiques (SC et $\mathrm{S}-\beta$ thal).Le taux compris entre 4-6g/dl a été le plus retrouvé soit une fréquence $52 \%$, suivi du taux 7 9g/dl. Nacoulma et al [18] trouvent chez les homozygotes, un taux d'hémoglobine compris entre 5,8 et $10,7 \mathrm{~g} / \mathrm{dl}$ avec une moyenne de 7,8 g/dl. Chez les doubles hétérozygotes SC, les taux variaient entre $6,6 \mathrm{~g} / \mathrm{dl}$ et $12,7 \mathrm{~g} / \mathrm{dl}$ avec une moyenne de 9,8 g /dl, Diagne [19] trouve chez des patients drépanocytaires un taux moyen d'Hb de base de $8,3 \pm 1,4 \mathrm{~g} / \mathrm{dl}$ chez les homozygotes, et de 10,9 $\pm 1,9 \mathrm{~g} / \mathrm{dl}$ chez 
les hétérozygotes composites SC. La présence d'infestation palustre chez $36 \%$ des malades dans notre contexte et l'absence de paludisme grave sont similaires à ceux observés en Côte d'Ivoire par A Sangaré et al. [13]. Dans leur étude sur la relation entre paludisme et drépanocytose, l'existence de l'infestation palustre a été prouvée de façon directe par la positivité de la goutte épaisse chez $39 \%$ des malades et par la présence d'accès palustres francs chez $24 \%$ des patients. Plasmodium falciparum a été l'espèce retrouvée chez tous nos malades présentant un paludisme; les autres espèces n'ont pas été identifiées. L'analyse des différents tableaux cliniques observés dans notre série a noté que des formes atténuées d'accès palustre; aucune forme grave n'a été notée. Ces résultats renforcent l'hypothèse que l'hémoglobine S protégerait contre les formes graves de paludisme.

Nous avons retrouvé sur les dix hémocultures positives différents germes
Salmonella enterica sérotype Typhi (4 fois), Staphylococcus aureus (1fois), Enterobacter cloacae (1fois); dont le plus rédoutable chez l'enfant drépanocytaire Streptococcus pneumoniae (4 fois). Selon Norris et al. [20] le Streptococcus pneumoniae est le germe le plus fréquent (42\%) suivi des bacilles Gram-négatifs (28\%). L'hémoculture a été stérile dans $52 \%$ des cas. La stérilité de l'hémoculture est certainement liée à l'utilisation d'antibiotiques avant l'hospitalisation car en cas de maladie chez le drépanocytaire au Mali les mères ont recours à l'automédication dans 18,3\% des cas [21].Nous avons été confrontés à la présence d'un germe nosocomial multi résistant (Enterobacter cloacae) chez un patient avec un issu défavorable.

\section{Localisation de l'infection selon le} résultat de l'hémoculture

Les salmonelles $(75 \%)$ et le staphylocoque (25\%) ont été isolés au cours des infections ostéoarticulaires. $\mathrm{Au}$ cours des ostéoarthrites de l'enfant dans le service de 
chirurgie pédiatrique de l'hôpital Gabriel

Toure Coulibaly et al [22] ont retrouvé le

Staphylocoque doré et Salmonella enterica

sérotype Typhi avec une fréquence de

$43,55 \%$ chacun chez les enfants drépanocytaires.

Dans la série de NACOULMA [23] les

Cocci Gram positif représentaient 52,5\%, les entérobactéries $42,5 \%$ et les bacilles Gram négatif et entérobactéries $5 \%$. La répartition par espèce montrait la prédominance de Staphylococcus aureus (42,5\%) suivi par Proteus mirabilis $(12,5 \%)$ et Escherichia coli $(7,5 \%)$. Au cours des infections pulmonaires le pneumocoque a été isolée chez un seul patient soit une fréquence de $25 \%$; Les hémocultures étaient stériles dans $75 \%$ des cas étudiés contrairement à Sangaré A.
[13], qui trouve Haemophilus influenzae, staphylocoques, streptocoques et pneumocoques.

Koffi. K .G et al [24] en s'intéressant aux causes bactériennes des infections respiratoires chez le drépanocytaire découvre que l'atteinte du parenchyme pulmonaire est notée dans $93 \%$ sous forme de pneumopathie contre $7 \%$ de bronchite aiguë.

Conclusion: Les complications infectieuses sont fréquentes chez l'enfant drépanocytaire au Mali. Elles ont été surtout rencontrées dans la tranche d'âge de 0-5ans. La disponibilité de l'hémoculture doit nous permettre de caractériser le profil bactériologique dans notre contexte.

\section{Références}

1- Haute Autorité De Santé de France .Recommandations cliniques dans Prise en charge de la drépanocytose chez l'enfant et l'adolescent, 2005.
2- Hau I, Coïc L. L’enfant drépanocytaire aux urgences. Pédiatrie 2008 ; 11(1) : 3542. 
3-Lesprit E, Philipe R. Prévention des infections chez l'enfant drépanocytaire

Pédiatre, Hôpital Trousseau, Paris.

Pédiatre, Hôpital Intercommunal, Créteil.

Développement et Santé, 2006; ${ }^{\circ} 182$.

4- Rapport OMS sur la Drépanocytose

Cinquante-neuvième assemblée mondiale de la sante.

5- Rochant H, Bernaudin F, Cornu G, Davies S, Fondu P, Galacteros F et al Place de la greffe de moelle dans le traitement de la drépanocytose. Hématologie 1996;2:334-43.

6- Tshilolo L. Les complications habituelles de la drépanocytose chez l'enfant en Afrique Centre Hospitalier Monkole / Centre de Formation et d'Appui Sanitaire (CEFA), Hôpital Général de Référence-ZS Mt NgafEula, RD Congo, Développement et Santé, 2006; n 182 .

7-OMS. La Drépanocytose dans la Région Africaine. Situation actuelle et perspective OMS ; Cinquante-sixième session Addis Abéba, Ethiopie, 28 août-1er septembre 2006.
8- Girot R. Thalassémie, drépanocytose. Rev Prat 1999; 49 : 667-74.

9- Anoosha H, Dora B, Godeau B. Complications aiguës de la drépanocytose. Rev prat, 2004; 54 (14) :1548-56.

10- Schmugge M, Speer O, Ozsahin, Gabriele M. La drépanocytose en Suisse: Physiopathologie, clinique. Forum Med Suisse 2008; 8: 582-6.

11- Nacoulma WC, Kam L, Gue E, Kafando E, Ayereroue J, Blot I. Évaluation du statut vaccinal de l'enfant drépanocytaire de la ville de Ouagadougou (Burkina Faso).Cahiers Santé 2006;16:155-9.

12- Gody J. C, Yanza M.C, Boka -Yao A, Mbombo F, Sepou A. Aspects actuels de la drépanocytose au complexe pédiatrique de Bangui (Centrafrique) : à propos de 123 cas. Med Afr Noire 2007;54:596-600.

13- Sangare A, Diop S, Koffi G, Ndahtz E, Allangba O, Aka Adjo Ma, Sanogo I. Profil infectieux chez le drépanocytaire, Bull Soc Pathol Exot 1997;90:339-41. 
14-Masmoudi A, Kitar A, Rebai M, Bouassida S, Turki H, Zahaf A. Causes d'hospitalisation des enfants drépanocytaires : influence de l'âge (C.H.U. de Brazzaville, Congo). Bull Soc Pathol Exot 2005;98:392-3.

15-Galacteros. Bases physiopathologiques de la drépanocytose, prise en charge et actualités thérapeutiques. Bull Soc Pathol Exot. 2001;94,(2):77-9.

16- Souna BS, Abarchi H, Karadji SA. Les ostéomyélites chez les drépanocytaires aspect : épidémiologique, diagnostique et thérapeutique .A propos de 66cas colligés à Niamey. Mali Med 2006;21(4):21-5.

17-Tekou H. A. Foly, B. Akue.

Le profil actuel des ostéomyélites hématogènes de l'enfant au CHU de Tokoin, LOME, TOGO. A propos de 145 Cas. Med Trop 2000;60:365-68.

18-Nacoulma EWC, Sakande J, Kafando

E, Kpowbie ED, Guissou IP. Profil hématologique et biochimique des drépanocytaires SS et SC en phase stationnaire au centre hospitalier national
Yalgado OUEDRAOGO de Ouagadougou. Mali Med, 2006; 21(1):8-11.

19-Diagne I, Diagne-Gueye, Signate-Sy. $\mathrm{H}$, et al. Prise en charge de la drépanocytose chez l'enfant en Afrique : expérience de la cohorte de l'hôpital ALBERT ROYER de Dakar. Med Trop 2003;63:513-20.

20-Norris FC, Smith KW, McGowan KL. Hémocultures positives dans la drépanocytose: le temps de positivité et le résultat clinique. Pediatr Oncol Hematol, 2003;25:390-532.

21- Sangho H, Dembele Keita H, Sidibe Keita A, Diarra F Y, Boureyma B, Dia A, et al. Enquête CAP des ménages sur la prise en charge de l'enfant drépanocytaire à Bamako, Mali Méd 2009;24(3):53-6.

22- Coulibaly Y, Keita M, Maiga AKM, Guindo Y, Alwata I, Toure AA. Ostéomyélite drépanocytaire au service d'Orthopédie et de Traumatologie du CHU Gabriel Touré. Mali Med 2010;25(4):2931. 
23- Nacoulma EWC, Bonkoungou P, 24- Koffi KG, Sangare A, Kone M, Dembelele D, Ye D, Kam L. Aspects Segbena Y. Etiologie des infections bactériologiques des infections ostéo- respiratoires basses dans la drépanocytose articulaires non tuberculeuses de l'enfant majeure du noir africain. Med Afr au centre hospitalier universitaire Sourô Noire 2001;48:333.

Sanou de Bobo-Dioulasso. Med Afr Noire 2011;58(1):36-40. 
Tableau I : répartition des enfants selon le diagnostic d'admission et formes drépanocytoses

\section{Formes}

\begin{tabular}{lcccc} 
Diagnostics & S- $\boldsymbol{\beta}$ thal & SC & SS & Total \\
\hline Accès palustre & 0 & 0 & 9 & 9 \\
Septicémie & 1 & 0 & 5 & 6 \\
Hépatite B & 0 & 0 & 1 & 1 \\
Pneumopathie & 1 & 0 & 3 & 4 \\
Cholécystite aigue & 0 & 0 & 1 & 1 \\
Ostéomyélite & 1 & 0 & 0 & 1 \\
Arthrite & 0 & 1 & 2 & 3 \\
Total & 3 & 1 & 19 & 25 \\
\hline
\end{tabular}

Tableau II : Répartition des germes selon la forme d'hémoglobinopathie

\begin{tabular}{lcccc}
\hline \multirow{2}{*}{ Germes } & Formes & & \\
\hline Salmonella enterica & SC & SS & Total \\
Typhi & 0 & 0 & 4 & 4 \\
Streptococcus pneumoniae & 1 & 0 & 3 & 4 \\
Staphylococcus aureus & 0 & 1 & 0 & 1 \\
Enterobacter cloacae & 1 & 0 & 0 & 1 \\
Total & 2 & 1 & 7 & 10 \\
\hline
\end{tabular}


Tableau III : Répartition des enfants en fonction de la pathologie associée et de l'évolution.

\begin{tabular}{lccc}
\hline Pathologies associées & \multicolumn{3}{c}{ Evolution } \\
& Favorable & Décès & Total \\
\hline Accès palustre & 9 & 0 & 9 \\
Septicémie & 3 & 3 & 6 \\
Hépatite B & 1 & 0 & 1 \\
Pneumopathie & 4 & 0 & 4 \\
Cholécystite aigue & 1 & 0 & 1 \\
Ostéomyélite & 1 & 0 & 1 \\
Arthrite & 3 & 0 & 3 \\
Total & 22 & 3 & 25 \\
\hline
\end{tabular}

